In Heft 32 der SÄZ erschien ein Beitrag von Otfried Höffe, in dem der Professor für Philosophie an der Universität Tübingen und Präsident der Nationalen Ethikkommission im Bereich Humanmedizin für eine prinzipienstarke und prinzipientreue philosophische Ethik plädiert*. Für eine andere Sichtweise steht Johannes Fischer, Professor für Theologische
Ethik und Leiter des Instituts für Sozialethik am Ethik-Zentrum der Universität Zürich, dessen Reflexionen nachfolgend wiedergegeben werden. Otfried Höffe nimmt auf Seite 1488 mit einer Replik Stellung.

Die Redaktion

\title{
Ethik als Prinzipienethik?
}

\section{Johannes Fischer}

Professor für Theologische Ethik, Leiter des Instituts für Sozialethik und verantwortlicher Leiter des universitären Forschungsschwerpunktes Ethik am Ethik-Zentrum der Universität Zürich
Bei einem wissenschaftlichen Anlass in Zürich wurde einem katholischen Moraltheologen aus Deutschland die Frage gestellt, was für sein ethisches Denken prägend gewesen ist. Seine Antwort war, dass dies die Erfahrung des Nationalsozialismus gewesen sei, den er als Kind und Jugendlicher erlebte. Diese Erfahrung habe ihn zu der tiefen Überzeugung geführt, dass es objektive und unverrückbare Massstäbe des Richtigen und Guten geben müsse, die es ermöglichen, Unrecht zweifelsfrei als Unrecht zu qualifizieren. Dementsprechend betrachtete er es als die Aufgabe der Ethik, diese Massstäbe aufzuzeigen und zu begründen.

Wie an dieser Antwort deutlich wird, gibt es gute Gründe für die Vermutung, dass die Differenzen, die Ethikerinnen und Ethiker in fundamentalethischen Fragen trennen, nicht selten auf Hintergrundüberzeugungen zurückzuführen sind, die mit persönlichen Prägungen zu tun haben und die im ethischen Diskurs kaum je ausdrücklich werden. Wie das Beispiel zeigt, sind sie zunächst einmal nicht mehr als ein Glaube, der die Richtung des ethischen Denkens bestimmt. Es verhält sich in solchen Fällen nicht so, dass sie aus der ethischen Reflexion selbst gewonnen worden wären. Vielmehr ist umgekehrt das aus ihnen resultierende ethische Denken darauf gerichtet, sich ihrer ex post zu vergewissern und ihnen eine rationale Grundlage zu geben. Solche Überzeugungen können auch die Erwartungen bestimmen, die sich öffentlich an die Ethik richten. halten, die es gerade im Blick auf die Medizinethik verdienen, näher betrachtet zu werden, nämlich erstens, dass die moralische Orientierung eine Orientierung an solchen Grundsätzen, d.h. an Urteilen über richtig oder falsch, gerecht oder ungerecht usw. ist, und zweitens, dass es in der Moral um das für jedermann Gültige geht, weshalb die Ethik die Aufgabe hat, die allgemeine bzw. universelle Geltung moralischer Grundsätze und Urteile zu begründen.

Betrachten wir zunächst die erste Unterstellung. Stimmt es, dass alles Handeln, dem wir eine moralische Qualität zumessen, an moralischen Urteilen über richtig oder falsch orientiert ist? Stellen wir uns vor, wir werden Zeugen eines Unfalls mit Verletzten. Ersichtlich ist hier der nächstliegende Grund dafür, warum wir helfen, die Tatsache, dass die Verletzten Hilfe brauchen, und nicht der Gedanke, dass es moralisch richtig oder geboten ist, ihnen Hilfe zu leisten. Wir orientieren uns also nicht an der moralischen Bewertung unseres Handelns. Vielmehr haben wir die Situation im Blick, die nach einem bestimmten Handeln verlangt, und sie ist es, die uns zu solchem Handeln veranlasst.

Angenommen, wir verpassen aufgrund der Hilfe für die Verletzten einen wichtigen Termin und müssen uns dafür rechtfertigen. Wir werden dann diese Situation als Grund nennen, indem wir sie dem anderen schildern, d. h. narrativ vor Augen führen, um ihn von der Richtigkeit, ja Gebotenheit unseres Handelns zu
* Höffe O. Philosophische Ethik: Fahne im Wind oder Fels in der Brandung? Schweiz Ärztezeitung. 2010;91(32):1199-1202.

Korrespondenz: Prof. Dr. theol. Johannes Fischer Institut für Sozialethik Ethik-Zentrum der Universität Zürich

Zollikerstr. 117

CH-8008 Zürich

fischer@sozethik.uzh.ch

\section{Stimmt es, dass alles Handeln, dem wir eine moralische Qualität zumessen, an moralischen Urteilen über richtig oder falsch orientiert ist?}

So sehr derartige Hintergrundüberzeugungen aufgrund ihres Anliegens Respekt abnötigen, so sehr ist doch zu fragen, ob sie den Blick auf das Phänomen der Moral und die Aufgabe der Ethik nicht eher verstellen. Sie weisen der Ethik die Aufgabe der rationalen, d.h. argumentativen Begründung fundamentaler moralischer Grundsätze oder Prinzipien zu, so dass jeder Vernünftige zu deren Anerkennung genötigt ist. In dieser Aufgabenbestimmung sind zwei Unterstellungen ent- überzeugen. Narrative vergegenwärtigen Situationen in ihrer moralischen Signifikanz. Narrative Gründe unterscheiden sich in einem wesentlichen Punkt von Argumenten, die bei der Auffassung von Ethik als rationaler Begründung der Moral im Zentrum stehen. Argumente sind Gründe, mit denen der Sprecher seinem Gegenüber nachweist, dass etwas richtig oder falsch ist, um solchermassen Zustimmung zu erzwingen. Hier liegt der Fokus auf der moralischen Bewertung, deren 
Gültigkeit nachgewiesen werden soll. Bei narrativen Gründen hingegen muss sich dies dem anderen selbst zeigen, indem er sich die Situation vor Augen führen lässt. Hier liegt der Fokus auf der Vergegenwärtigung der moralischen Signifikanz einer Situation. Im Unterschied zu der Nötigung, die vom zwanglosen Zwang eines Arguments ausgeht, geht es hier um eine Nötigung, die von einer gegebenen Situation ausgeht und der wir uns aufgrund von deren Anschauung nicht entziehen können [1].
In vielen Fällen halten wir uns mit Behauptungen zurück, weil wir uns nicht in der Lage sehen, den damit verbundenen diskursiven Anspruch einzulösen. Aber wir verzichten deshalb nicht auf ein eigenes Urteil, z. B. in einer strittigen moralischen Frage. Was hier für Irritationen sorgen kann, das ist, dass nicht unterschieden wird zwischen dem Anspruch, den wir mit einem Urteil erheben und der sich nach dem Gesagten auf dessen Wahrheit, nicht aber auf dessen Geltung für andere bezieht, und der Reichweite der Aussage, für

\section{Deshalb ist es so wichtig zu sehen, dass wir mit moralischen Urteilen keinen Anspruch}

\section{auf Geltung für andere, d.h. auf allgemeine Geltung erheben}

Es ist die Vorstellung, dass die moralische Orientierung eine Orientierung an moralischen Bewertungen ist, die zu der Meinung führt, dass die Ethik ihre Aufgabe in der Begründung moralischer Bewertungen hat. Hieraus bezieht das prinzipienethische Denken seine vordergründige Plausibilität, indem es allgemeine Grundsätze für moralische Bewertungen aufzustellen sucht. Doch liegt darin eine problematische Verschiebung des Fokus der moralischen Orientierung. Man stelle sich eine Ethikkommission vor, die eine Stellungnahme zur Suizidbeihilfe abgeben soll. Wenn die moralische Orientierung eine Orientierung an moralischen Bewertungen wäre, dann müsste man es als deren Aufgabe betrachten, ein Urteil über die moralische Richtigkeit oder Falschheit der Suizidbeihilfe abzugeben, um solchermassen zur öffentlichen Orientierung in dieser Frage beizutragen. Anders verhält es sich, wenn die moralische Orientierung ihre Gründe aus der Anschauung der konkreten Fälle bezieht, an die hier zu denken ist. In diesem Fall besteht die Aufgabe der Kommission darin, ein öffentliches Bewusstsein zu schaffen für die Situation derer, um die es in dieser Frage geht.

Betrachten wir nun die zweite Unterstellung, wonach moralische Urteile mit einem Anspruch auf allgemeine Geltung verbunden sind, woraus für die Ethik abgeleitet wird, dass sie die Allgemeingültigkeit moralischer Urteile zu begründen hat. Ersichtlich beruht diese Auffassung auf einer Verkennung des Anspruchs, der mit Urteilen erhoben wird. Mit Urteilen erheben wir lediglich einen Anspruch auf Wahrheit, der sich auf das Verhältnis von Aussage und Sachverhalt bezieht. Allgemeine Geltungsansprüche erheben wir demgegenüber mit Behauptungen oder Thesen. Mit ihnen übernehmen wir die diskursive Verpflichtung, den Erweis für das Behauptete zu erbringen, und dies geschieht in Gestalt von Argumenten. Für einen solchen Erweis sind narrative Gründe zu schwach, da ihre Überzeugungskraft kontingent davon abhängt, ob sich dem anderen die moralische Signifikanz einer Situation in derselben Weise vor Augen stellt wie uns selbst. die dieser Anspruch erhoben wird und die das Handeln anderer mit umfasst, und so ergibt sich das Missverständnis, dass moralische Urteile einen Anspruch auf Allgemeingültigkeit erheben.

In Lehrbüchern der Ethik wird diese für das Verständnis der Moral wichtige Unterscheidung zwischen Urteilen und Behauptungen zumeist nicht gemacht, und so hält sich hartnäckig die Meinung, dass der Anspruch auf Allgemeingültigkeit zu den Wesensmerkmalen moralischer Urteile gehört [2]. Doch ist es ersichtlich zweierlei zu sagen: «Beihilfe zum Suizid ist moralisch falsch», oder zu sagen: «Ich behaupte, dass die Beihilfe zum Suizid moralisch falsch ist.» Mit der zweiten Äusserung, der Behauptung, erheben wir einen diskursiven Anspruch auf allgemeine Geltung, mit der ersten Äusserung, dem Urteil, hingegen nicht. Das schliesst nicht aus, dass wir gleichwohl Gründe vorbringen werden, wenn man uns fragt. Aber diese haben nicht die Gestalt und den Anspruch von Argumenten.

Dies führt zu der grundsätzlichen Frage, ob sich Aussagen moralischen Inhalts überhaupt zum Gegenstand von Behauptungen machen lassen, was bedeuten würde, dass sich für sie ein argumentativer Nachweis führen lassen müsste. Die Antwort hängt davon ab, zu welcher Auffassung man hinsichtlich der seit der Antike erörterten Frage nach den Grundlagen der Moral gelangt. In der heutigen Moralphilosophie gibt es hierzu eine intensive Debatte, die sich vor allem auf die Bedeutung von Emotionen für die moralische Orientierung bezieht [3]. Sie konvergiert in wesentlichen Punkten mit den Erkenntnissen der heutigen empirischen Moralforschung in Psychologie und Neurobiologie, wonach moralische Wertungen auf der emotionalen Bewertung von Situationen und Handlungen beruhen [4]. Dies entzieht der Vorstellung jede Grundlage, dass die Wahrheit moralischer Urteile einem anderen argumentativ andemonstriert werden kann. Sie kann sich dem anderen nur selbst zeigen, indem er sich die betreffende Situation oder Handlung vor Augen führt und sie dabei emotional bewertet. Des- 
halb ist es so wichtig zu sehen, dass wir mit moralischen Urteilen keinen Anspruch auf Geltung für andere, d.h. auf allgemeine Geltung erheben. Von hier fällt nicht zuletzt Licht auf die Bedeutung der Narrativität für die Moralreflexion. Sie besteht darin, dass Narrative moralische Sachverhalte für die emotionale Erkenntnis vergegenwärtigen, indem sie Situationen und Handlungen in ihrer Erlebnisqualität vor das innere Auge stellen.

Dieser Punkt ist gerade für die medizinische Ethik von Bedeutung. Vor Jahren fand am Ethik-Zentrum der Universität Zürich eine Tagung statt mit dem Titel «Potentiale philosophischer Medizinethik», die so etwas wie eine Standortbestimmung der philosophischen Medizinethik versuchte. Das Thema, das hierfür zur Illustration diente, war die Sterbehilfe. Es zeigte sich im Verlauf der Tagung eine auffällige Diskrepanz. Das Interesse der Philosophinnen und Philosophen wandte sich sehr schnell Prinzipien- und Begründungsfragen der Medizinethik zu. Ein Nachmittag war den Medizinern reserviert. Besonders ein Vortrag hinterliess bei den anwesenden Nichtmedizinern einen tiefen Eindruck, und zwar einfach dadurch, dass der Vortragende sich darauf beschränkte, von Fällen von Sterbebegleitung zu erzählen, mit denen er in seiner Tätigkeit als Arzt zu tun hatte. Es fehlte in seinen Ausführungen jede Berufung auf moralische Prinzipien. Allein die narrative Präsentation von Fällen von Sterbebegleitung und Sterbehilfe machte deutlich, dass er werden sollen und woher sie ihre Geltung beziehen. Jedenfalls kann nicht die Tatsache, dass sie faktisch innerhalb einer bestimmten Gruppe wie z.B. der Ärzteschaft anerkannt werden und in Geltung stehen, massgebend hierfür sein, da die normative Geltung moralischer Normen nicht aus ihrer faktischen Geltung abgeleitet werden kann. Was faktisch in Geltung steht, kann auch moralisch falsch sein. Angesichts der Grenzen, an die jede Spezifizierung von Regeln stösst, stellt sich überdies die Frage, wie man je vom Prinzip zum konkreten Einzelfall gelangen kann. Diese Probleme stellen sich nicht im zweiten Fall, bei dem das Prinzip über die Generalisierung der Anschauung der moralischen Signifikanz konkreter Situationen gewonnen wird. So gewinnt die Norm, dass Menschen nicht erniedrigt werden dürfen, ihre Evidenz aus der Vergegenwärtigung entsprechender Szenarien unter der Fragestellung, was es für einen Menschen bedeutet, erniedrigt zu werden. Wenn Prinzipien ihre normative Geltung und ihren Gehalt auf diese Weise beziehen, dann muss sich die Ethik gerade für die konkreten Fälle und Situationen interessieren, statt ihren Standpunkt auf einer prinzipiellen Ebene zu nehmen, wie dies die Auffassung vieler Ethiker ist. Die orientierende Kraft von Regeln wie z. B. medizinethischen Richtlinien liegt dann darin, dass sich in ihnen die Anschauung und Erfahrung von vielen Situationen verdichtet und dass sie solchermassen einschärfen, worauf bei einer Entscheidung zu achten ist.

\section{Die orientierende Kraft von Regeln wie z. B. medizinethischen Richtlinien liegt dann darin, dass sich in ihnen die Anschauung und Erfahrung von vielen Situationen verdichtet}

als Arzt in diesen Fällen so handeln musste, wie er gehandelt hat, und dass dies auch in einem moralischen Sinne angemessen und richtig war. Im Anschluss an den Vortrag entzündete sich eine Debatte darüber, ob das Vorgetragene irgendetwas mit Ethik zu tun hatte, was von der Mehrzahl der Philosophinnen und Philosophen verneint wurde. Nach ihrer Sicht war die Ethik erst bei ihrer Sache, wenn sie sich mit moralischen Prinzipien und deren Begründung befasst.

Nun wäre das bisher Ausgeführte allerdings missverstanden, würde man daraus schliessen, dass Regeln oder Prinzipien überhaupt keine Bedeutung für die Moral zukommt. Die entscheidende Frage ist, was zuerst ist: das Prinzip, das dann vor die Aufgabe stellt, es in Richtung auf konkrete Situationen zu spezifizieren, oder umgekehrt die Anschauung der moralischen Signifikanz konkreter Situationen, die dann zu einer Regel oder einem Prinzip generalisiert wird. Wenn Ersteres der Fall sein soll, dann stellt sich die Frage, woher die Prinzipien - etwa die vier medizinethischen Prinzipien von Beauchamp und Childress - genommen
Ich kehre damit zum Anfang zurück, d.h. zu den Hintergrundüberzeugungen bzw. dem Glauben von Ethikerinnen und Ethikern, dass es doch objektive und unverrückbare moralische Grundsätze geben muss, die aufzuzeigen und rational zu begründen Aufgabe der Ethik ist. Ich habe einige Gründe genannt, die diesen Glauben und die damit verbundenen Erwartungen an die Ethik in Zweifel ziehen. In historischer Perspektive muss man überdies sehen, dass die Ethik erst in der Moderne unter den Zweck der rationalen, d.h. argumentativen Begründung der Moral gestellt worden ist. Die modernen ethischen Theorien wie der Kantianismus und der Utilitarismus beanspruchen, eine solche Begründung zu liefern. Die antike Ethik hat diesen Zweck und somit auch derartige Theorien nicht gekannt [5]. Diese Zwecksetzung gibt dem modernen ethischen Denken sein besonderes Gepräge. Charakteristisch für dieses Denken ist die Haltung des Desengagements. Desengagement hat es mit der Objektivierung eines Bereichs zu tun, durch die diesem die normative Kraft genommen wird, mit der er auf uns wirkt 
[6]. In dem Beispiel des Unfalls mit Verletzten ist es die moralische Signifikanz der erlebten Situation, die uns zum Handeln veranlasst. Sie hat, wie gesagt, ihre sprachliche Artikulation in einem Narrativ. Geht es hingegen nach dem die moderne Ethik beherrschenden Rationalitätsideal, dann zählt nicht, wie eine Situation erlebt wird oder wie sie sich aufgrund eines Narrativs vor Augen stellt, da dies von kontingenten Bedingungen abhängt, sondern es zählen allein Argumente. Situationen werden dabei zu Anwendungsfällen von Prinzipien bzw. Regeln, und hierfür ist ihre deskriptive, beschreibende Charakterisierung massgebend, im Unterschied zu ihrer narrativen Vergegenwärtigung. Rein deskriptiv aufgefasst büssen sie ihre normative Wirkung auf uns ein. Die Folge dieser Objektivierung ist der Hiatus zwischen (deskriptiven) Tatsachen und Wertungen, der für das moderne ethische Denken charakteristisch ist und auf dem ein Phänomen wie der naturalistische Fehlschluss beruht. In
Laufen diese Überlegungen auf einen ethischen Relativismus hinaus? Unter Relativismus versteht man in der Regel eine Auffassung, die den Wahrheitsanspruch, den wir mit moralischen Urteilen erheben, auf die eigene Gruppe oder Kultur einschränkt. Doch wenn wir urteilen, dass die Erniedrigung oder Folterung von Menschen verwerflich ist, dann sagen und meinen wir nicht, dass dies für uns verwerflich ist, sondern wir sagen, dass dies verwerflich ist, wo auch immer dies geschieht. Dazu steht die Einsicht nicht in Widerspruch, dass unsere moralischen Auffassungen durch die Geschichte unserer Kultur geprägt sind und dass sie keineswegs universal sind. So beruht eine archaische Ehrenmoral, wie sie in vielen Gesellschaften bis in die Gegenwart anzutreffen ist, auf anderen emotionalen Einstellungen, als sie unter dem Einfluss des Christentums ausgebildet worden sind. Gerade das Bewusstsein des geschichtlichen Gewordenseins moralischer Einstellungen sowie die Einsicht, dass die mora-

\section{Eine Moralphilosophie, die uns von der Befangenheit in vermeintlich irrationalen Emotionen befreien möchte, gleicht dem freundlichen Affen, der einen Fisch, um inn vor dem Ertrinken zu retten, sicher auf einen Baum setzt}

unserer lebensweltlichen Erfahrung gibt es diesen Hiatus nicht, wie das Beispiel des Unfalls zeigt, bei dem in eins mit der narrativen Vergegenwärtigung der betreffenden Situation zugleich die Richtigkeit des betreffenden Handelns vor Augen gestellt wird.

Hier dürfte die Erklärung liegen für die Irritationen, die die professionelle Ethik häufig bei ethischen Laien auslöst. Deren moralische Urteile und Überzeugungen resultieren aus der Anschauung der moralischen Signifikanz von lebensweltlichen Situationen, und sie sind dabei emotional engagiert. Für grosse Teile der heutigen Ethik ist jedoch gerade dieses emotionale Engagement verdächtig und irrtumsanfällig. Moralische Wahrheit verbürgt nach dieser Sicht allein ein desengagierter Standpunkt, der sich der rationalen Begründung von moralischen Grundsätzen und Urteilen verpflichtet weiss. Freilich, wenn die heutige Moralforschung mit ihrer Einsicht in die emotionalen Grundlagen der Moral recht hat, dann hat ein solcher Standpunkt mit Moral wenig zu tun. Eine Moralphilosophie, die uns von der Befangenheit in vermeintlich irrationalen Emotionen befreien möchte, gleicht dem freundlichen Affen, der einen Fisch, um ihn vor dem Ertrinken zu retten, sicher auf einen Baum setzt. Sie isoliert uns von etwas Entscheidendem, das zu unserer Konstitution als moralische Subjekte gehört. lische Sensibilität, wenn sie abstumpft oder verroht, nicht durch rationale Überlegungen und Argumente wiederhergestellt werden kann, werden uns umso stärker dafür besorgt sein lassen, solchen Abstumpfungstendenzen entgegenzutreten.

\section{Literatur}

1 Vgl. zu dieser Art der Nötigung Winch P. Wer ist mein Nächster? In: Ders. Versuchen zu verstehen. Frankfurt; 1992. S. 213-30.

2 Birnbacher D. Analytische Einführung in die Ethik. Berlin; 2003. S. 24-31.

3 Diese Debatte wird z.B. aufgearbeitet in: Ammann C. Emotionen - Seismographen der Bedeutung. Ihre Relevanz für eine christliche Ethik. Stuttgart; 2007.

4 Vgl. Fischer J. Grundlagen der Moral aus ethischer Perspektive und aus der Perspektive der empirischen Moralforschung. In: Ders., Gruden S. Die Struktur der moralischen Orientierung. Interdisziplinäre Perspektiven. Berlin; 2010. S. 19-48.

5 Vgl. dazu Tugendhat E. Antike und moderne Ethik. In: Ders. Probleme der Ethik. Stuttgart; 1984. S. 33-56. Tugendhat spricht von der «Radikalisierung des Begründungsgedankens» (aaO. S. 41) in der Moderne.

6 Vgl. Taylor C. Quellen des Selbst. Frankfurt;1996. S. 290 . 